\title{
Effects of IgG anti-GM1 monoclonal antibodies on neuromuscular transmission and calcium channel binding in rat neuromuscular junctions
}

\author{
SAYAKO HOTTA $^{1}$, YOSHIHIKO NAKATANI ${ }^{1}$, TOSHIE KAMBE ${ }^{2}$, KENJI ABE $^{3}$, \\ YUTAKA MASUDA $^{4}$, IKU UTSUMOMIYA ${ }^{5}$ and KYOJI TAGUCHI ${ }^{1}$
}

\begin{abstract}
Departments of ${ }^{1}$ Medicinal Pharmacology and ${ }^{2}$ Pharmacology, Showa Pharmaceutical University, Machida, Tokyo 194-8543;
${ }^{3}$ Department of Pharmacology, School of Pharmaceutical Sciences, Ohu University, Koriyama, Fukushima 963-8611;

${ }^{4}$ Laboratory of Clinical Pharmacy; ${ }^{5}$ Department of Developmental Education,

Showa Pharmaceutical University, Machida, Tokyo 194-8543, Japan
\end{abstract}

Received September 5, 2014; Accepted May 1, 2015

DOI: $10.3892 / \mathrm{etm} .2015 .2575$

\begin{abstract}
Guillain-Barré syndrome is a type of acute inflammatory neuropathy that causes ataxia and is associated with the IgG anti-GM1 antibody. However, the pathogenic role of the IgG anti-GM1 antibody and calcium channels in neuromuscular junctions (NMJs) remains unclear. Thus, the aim of the present study was to investigate the effects of the $\operatorname{IgG}$ anti-GM1 monoclonal antibody (mAb) on spontaneous muscle action potentials (SMAPs), and the effects of calcium channel blockers, in a rat spinal cord-muscle co-culture system. In addition, the binding of IgG anti-GM1 mAb to calcium channels was investigated in the rat hemidiaphragm. The frequency of SMAPs in the innervated muscle cells was acutely inhibited by the IgG anti-GM1 mAb; however, this effect was blocked by the N-type calcium channel blocker, $\omega$-conotoxin GVIA (30 nM). Furthermore, the P/Q-type calcium channel blocker, $\omega$-agatoxin IVA $(10 \mathrm{nM})$, was found to partially block the IgG anti-GM1 mAb-induced inhibitory effect in the spinal cord-muscle co-culture system. Immunohistochemical analysis of the rat hemidiaphragm indicated that IgG anti-GM1 mAb binding overlapped with anti-Ca $\mathrm{Ca}_{\mathrm{v}} 2.2\left(\alpha_{1 \mathrm{~B}}\right)$ antibody binding in the nerve terminal. In addition, IgG anti-GM1 $\mathrm{mAb}$ binding partially overlapped with anti-Ca $2.1\left(\alpha_{1 \mathrm{~A}}\right)$ antibody binding. Thus, the results demonstrated that the IgG anti-GM1 mAb binds to calcium channels in the nerve terminals of NMJs. Therefore, the inhibitory effect of IgG anti-GM1 mAb on
\end{abstract}

Correspondence to: Professor Kyoji Taguchi, Department of Medicinal Pharmacology, Showa Pharmaceutical University, 3-3165 Higashi-tamagawagakuen, Machida, Tokyo 194-8543, Japan E-mail: taguchi@ac.shoyaku.ac.jp

Key words: Guillain-Barré syndrome, IgG anti-GM1 monoclonal antibody, muscle action potential, calcium channel, neuromuscular junctions
SMAPs may involve N-type and P/Q-type calcium channels in motor nerve terminals at the NMJ.

\section{Introduction}

The motor nerve terminal has been proposed as a potential target for the binding of anti-ganglioside antibodies in Guillain-Barré syndrome (GBS) $(1,2)$. In cases of axonal GBS, autoantibodies against the ganglioside-like molecules, GM1, GM1b, GD1a and GalNAc-GD1a, are frequently detected, and these gangliosides may be targeted by autoantibodies. Anti-ganglioside antibodies are hypothesized to mediate the pathogenic mechanism of GBS, leading to axonal neuropathy.

Voltage-dependent calcium channels (VDCCs) serve a key function in neuromuscular transmission by facilitating calcium ion influx into motor nerve terminals, thereby affecting acetylcholine release. Previous studies have reported that anti-ganglioside antibodies induce alterations in calcium channels at neuromuscular junctions (NMJs) $(3,4)$. In addition, an IgG anti-GM1 monoclonal antibody ( $\mathrm{mAb}$ ) has been previously demonstrated to reduce the rate of spontaneous muscle action potential (SMAPs) in spinal cord-muscle co-culture systems and the VDCC current in cerebellar Purkinje cells $(5,6)$. Lipid microdomains, which contain gangliosides, are involved in the clustering of P/Q-type VDCCs and the organization of presynaptic membrane sites for synaptic exocytosis $(7,8)$. In a previous study, IgG anti-GalNAc-GDla antibodies, purified from a rabbit immunized with GalNAc-GDla, were shown to inhibit VDCC currents in differentiated PC12 pheochromocytoma cells (9). Collectively, these observations indicate that the inhibition of nerve conduction in patients with GBS may be associated with the dysfunction of VDCCs in motor nerve terminals.

In the present study, the effects of an IgG anti-GM1 mAb on SMAPs were evaluated using pretreatment with $\mathrm{N}$-type and P/Q-type calcium channel blockers in a rat spinal cord-muscle co-culture system. Immunohistochemical analysis using an IgG anti-GM1 mAb, calcium channel antibodies and triple fluorescence labeling were used to show the colocalization of 
IgG anti-GM1 mAb and calcium channels in the NMJs of the rat hemidiaphragm.

\section{Materials and methods}

IgG anti-GM1 $m A b$. An IgG anti-GM1 mAb was provided by Dr Nobuhiro Yuki (Departments of Microbiology and Medicine, National University of Singapore, 2 Science Drive, Singapore 17597 ), and all samples were stored at $-80^{\circ} \mathrm{C}$ until required. Using an enzyme-linked immunosorbent assay, reactivity was detected with the IgG anti-GM1 mAb, which was in accordance with previous observations by Hotta et al (5).

Drugs. Drugs were dissolved in the co-culture medium bathing the preparation. $\omega$-conotoxin GVIA and $\omega$-agatoxin IVA (Alomone Labs Ltd., Jerusalem, Israel) were dissolved in a stock solution containing cytochrome $c(1 \mathrm{mg} / \mathrm{ml}$; Sigma-Aldrich; St. Louis, MO, USA) to prevent the non-specific binding of the peptide to the chamber walls and tubing.

Spinal cord-muscle co-culture. Pregnant Wistar rats $(n=35)$ were purchased from Japan Laboratory Animals, Inc. (Tokyo, Japan) individually housed under controlled conditions, with a 12-h light-dark cycle and free access to food and water. Experiments were conducted in accordance with the Guidelines for Animal Care of Showa Pharmaceutical University (Tokyo, Japan), in addition to the guidelines for animal use published by the National Institutes of Health (https://grants.nih. gov/grants/olaw/Guide-for-the-Care-and-use-of-laboratory-animals. pdf). This study was conducted with the approval of the Showa Pharmaceutical University Research Ethics Committee (approval no. H18).

A spinal cord-muscle co-culture system was established according to a previously described method by Taguchi et al (10). Briefly, muscle was extracted from prenatal day 17 fetal rats and separated into $\sim 3-\mathrm{mm}$ sections in Tyrode's solution (Sigma-Aldrich) containing $100 \mu \mathrm{g} / \mathrm{ml}$ streptomycin and $100 \mu \mathrm{g} / \mathrm{ml}$ penicillin. The sections were subsequently incubated at $37^{\circ} \mathrm{C}$ for $20 \mathrm{~min}$ in $\mathrm{Ca}^{2+}$ - and $\mathrm{Mg}^{2+}$-free Tyrode's solution containing $1 \mathrm{mg} / \mathrm{ml}$ collagenase. For the innervation experiments, explants of the entire transverse slices of fetal spinal cord, including the dorsal root ganglia, were placed onto a collagen-coated 35-mm Petri dish. Individual muscle cells were derived from trituration and placed on the slices of spinal cord for culture. The muscle cells and spinal cords were co-cultured in 67\% DMEM (Gibco Life Technologies, Carlsbad, CA, USA), 23\% medium 199 (Gibco Life Technologies) and 10\% fetal calf serum (Roche Diagnostics, Basel, Switzerland), which was supplemented with $25 \mathrm{ng} / \mathrm{ml}$ fibroblast growth factor (Sigma-Aldrich) and $20 \mu \mathrm{g} / \mathrm{ml}$ insulin (Gibco Life Technologies). The spinal cord-muscle co-culture systems were stored in a $\mathrm{CO}_{2}$ incubator with $5 \% \mathrm{CO}_{2}$ and $95 \%$ $\mathrm{O}_{2}$ at $37^{\circ} \mathrm{C}$.

Measurement of SMAPs. After 1 week of co-culture, the innervated muscle specimens were placed in an experimental chamber on the stage of an inverted microscope (IX-70; Olympus Corporation, Tokyo, Japan). The 1-ml experimental chamber was continuously perfused with medium $(67 \%$ DMEM and 23\% medium 199) at a rate of $1-2 \mathrm{ml} / \mathrm{min}$, with continuous bubbling of $5 \% \mathrm{CO}_{2} / 95 \% \mathrm{O}_{2}$. Glass microelectrodes (GD-1; Narishige Group, Tokyo, Japan) filled with $3 \mathrm{M}$ $\mathrm{KCl}$, with a tip resistance of 20-40 $\mathrm{M} \Omega$, were used to record the SMAPs. Each electrode that was connected to a microelectrode amplifier (MEZ-8301; Nihon Koden Corporation, Tokyo, Japan) recorded the electrical activity, which was displayed on an oscilloscope (VC-11; Nihon Koden Corporation). After the stability of muscle action potentials was observed for $\sim 4 \mathrm{~min}$, $10 \mu \mathrm{l}$ anti-GM1 mAb antibody was applied using a micropipette. Data were transferred to and stored in a computer, using pCLAMP6 software (Molecular Devices, Sunnyvale, CA, USA). All experiments were conducted at $33 \pm 1^{\circ} \mathrm{C}$.

Immunohistochemical analysis. Hemidiaphragms were used for triple fluorescence labeling to determine the localization of GM1. Tissue samples were incubated in $10 \%$ normal goat serum (NGS; Funakoshi Co., Ltd., Tokyo, Japan) in Block Ace blocking agent (Dainippon Sumitomo Pharma Co., Ltd., Tokyo, Japan) for $30 \mathrm{~min}$ at room temperature to block non-specific binding, as previously described (6). After blocking, the tissues were incubated for $5 \mathrm{~h}$ at $4^{\circ} \mathrm{C}$ with IgG anti-GM1 antibodies (1:100; provided by Dr Nobuhiro Yuki) in 10\% NGS and $10 \%$ Block Ace in phosphate-buffered saline (PBS). In order to detect the IgG anti-GM1 mAb, the tissues were incubated for $1 \mathrm{~h}$ at $4^{\circ} \mathrm{C}$ with a fluorescein isothiocyanate (FITC)-conjugated anti-mouse IgG (1:100; Sigma-Aldrich). Subsequently, the hemidiaphragms were incubated with primary anti-Ca 2.1 $\left(\alpha_{1 \mathrm{~A}} ; 1: 1,000 ;\right.$ \#ACC-001) and anti-Ca $2.2\left(\alpha_{1 \mathrm{~B}} ; 1: 1,000\right.$; \#ACC-002) antibodies (Alomone Labs Ltd., Jerusalem, Israel) for $5 \mathrm{~h}$ at $4^{\circ} \mathrm{C}$. To detect the primary antibodies, the hemidiaphragms were incubated for $1 \mathrm{~h}$ at $4^{\circ} \mathrm{C}$ with a monoclonal Cy5-conjugated anti-rabbit IgG (1:100; AP182SA6; Chemicon International, Inc., Temecula, CA, USA). Hemidiaphragms were subsequently incubated with rhodamine- $\alpha$-bungarotoxin $(\alpha$-BuTx; 1:300; Molecular Probes; Invitrogen Life Technologies, Grand Island, NY, USA) for $5 \mathrm{~h}$ at $4^{\circ} \mathrm{C}$. Each reaction was terminated by numerous washes with $\mathrm{PBS}$, and the tissue was mounted using Aqua Poly/Mount (PolySciences, Inc., Warrington, PA, USA). All the immunostained sections were observed under an Olympus laser-scanning confocal microscope (Fluoview BW50; Olympus Corporation, Tokyo, Japan) at wavelengths of $488 \mathrm{~nm}$ (FITC), $543 \mathrm{~nm}$ (rhodamine) or $568 \mathrm{~nm}(\mathrm{Cy} 5)$.

Statistical analysis. Data are expressed as the mean \pm standard error of the mean (SEM). A paired t-test was used to compare the effects of the IgG anti-GM1 mAb, where $\mathrm{P}<0.05$ was considered to indicate a statistically significant difference. Statistical analyses were conducted using Microsoft Excel and statistical add-on software (Microsoft Corporation, Redmond, WA, USA).

\section{Results}

Effects of IgG anti-GM1 mAb on SMAPs. After 5-7 days of co-culture, asynchronous contraction of numerous individual muscle fibers was observed at the newly developed NMJs. SMAPs within the innervated muscle cells were recorded with a frequency of $12.7 \pm 4.8 \mathrm{sec}$ per $5 \mathrm{sec}$ and an amplitude of $65.4 \pm 8.4 \mathrm{mV}(\mathrm{n}=8$, mean \pm SEM $)$. IgG anti-GM1 mAb 
A

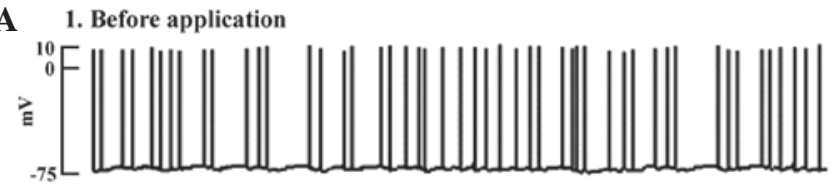

2. Anti-GM1 mAb (1:100) after 3 min

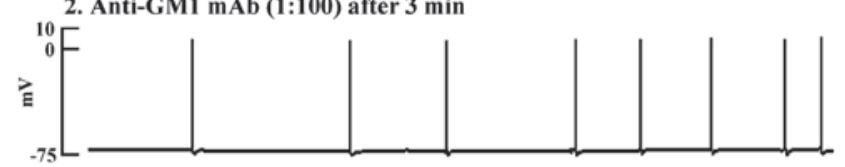

3. Wash out after $4 \mathrm{~min}$

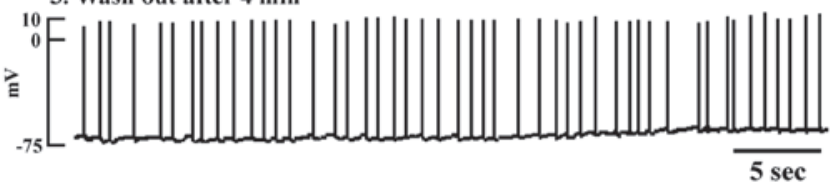

B

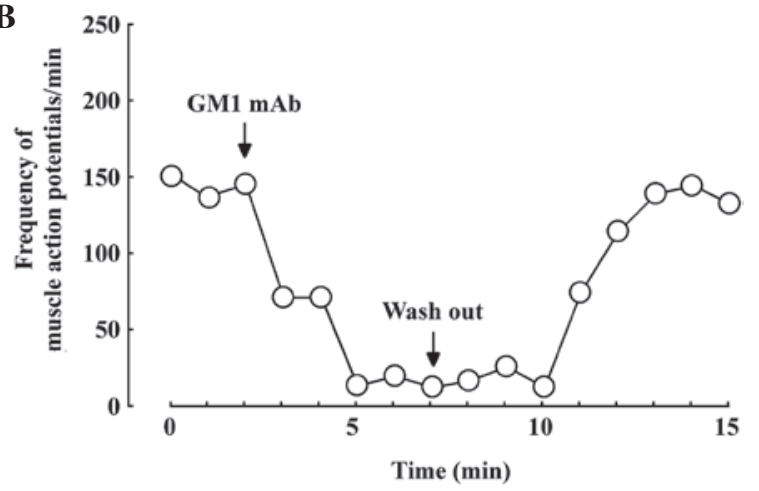

C

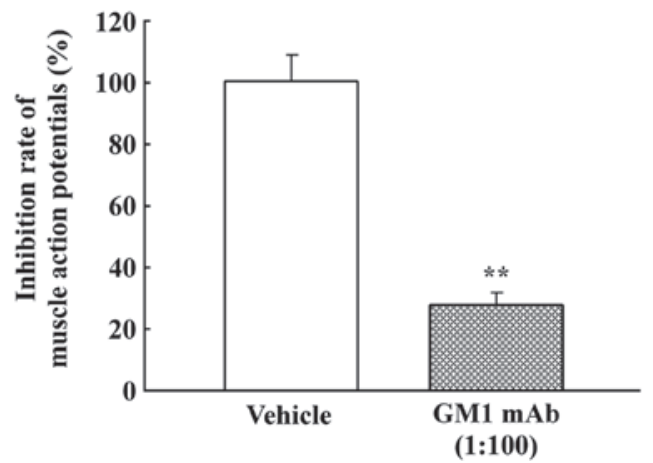

Figure 1. Effects of IgG anti-GM1 mAb on spontaneous muscle action potentials (SMAPs) in the spinal cord-muscle co-cultures. (A) Inhibition of SMAPs by IgG anti-GM1 mAb (1:100) in the spinal cord-muscle co-cultures, which was fully reversed after 4 min of washing with the co-culture medium. (B) Time course of the frequency of SMAPs following the addition of IgG anti-GM1 mAb. IgG anti-GM1 mAb decreased the SMAPs in the spinal cord-muscle co-cultures. After washing out the IgG anti-GM1 mAb, recovery from inhibition was observed. The number of SMAPs was measured every 5 sec. (C) Inhibition rate of SMAPs following the addition of IgG anti-GM1 mAb. Data are expressed as the mean \pm standard error of the mean $(\mathrm{n}=4$ for IgG anti-GM1 $\mathrm{mAb}){ }^{* *} \mathrm{P}<0.01$, vs. vehicle. $\mathrm{mAB}$, monoclonal antibody.

A
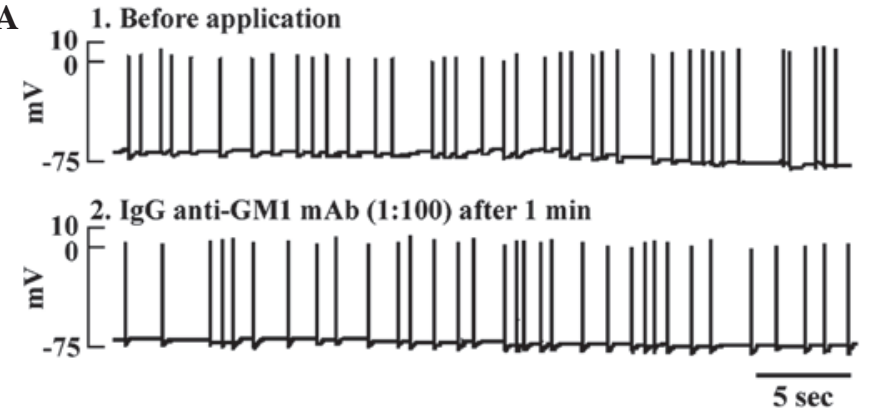

C
B

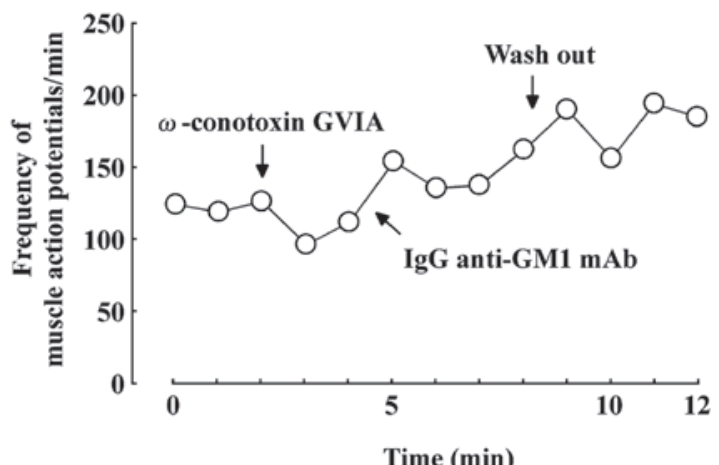

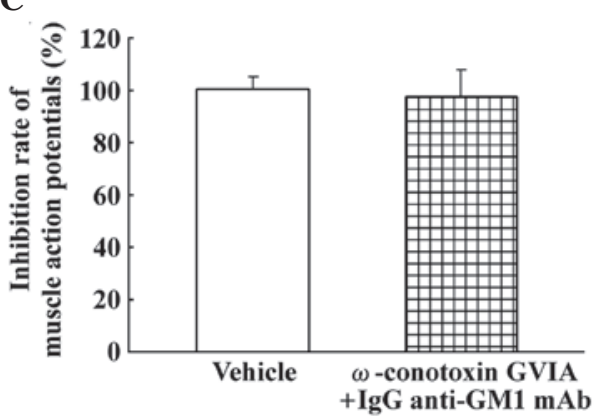

Figure 2. Effects of pretreatment with the N-type calcium channel blocker, $\omega$-conotoxin GVIA, on the inhibitory effect of IgG anti-GM1 mAb (1:100) on spontaneous muscle action potentials (SMAPs) in the spinal cord-muscle co-culture system. (A) Inhibition of SMAPs by pretreatment with $\omega$-conotoxin GVIA $(30 \mathrm{nM})$ in the spinal cord-muscle co-cultures. (B) Time course showing the reversal of the inhibitory effect on SMAPs mediated by IgG anti-GM1 mAb by pretreatment with $\omega$-conotoxin GVIA $(30 \mathrm{nM})$. The number of SMAPs was measured every 5 sec. Arrows show the time of application of $\omega$-conotoxin GVIA, IgG anti-GM1 mAb and the exchange of the co-culture medium. (C) Inhibition rate of SMAPs following the addition of IgG anti-GM1 mAb. Error bars represent the mean \pm standard error of the mean of data pooled from 4-6 spinal cord-muscle co-cultures. $\mathrm{mAB}$, monoclonal antibody. 
A

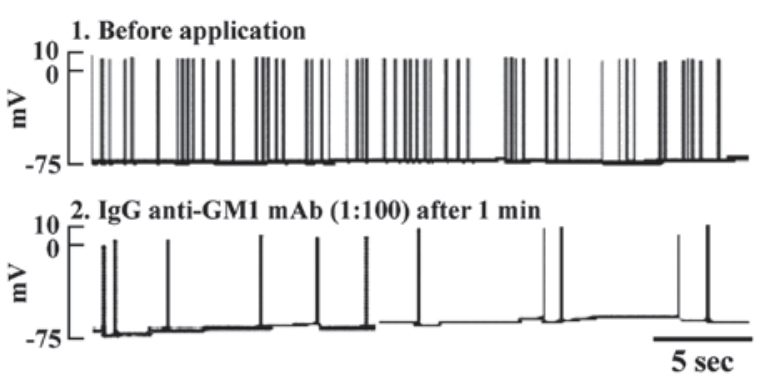

B

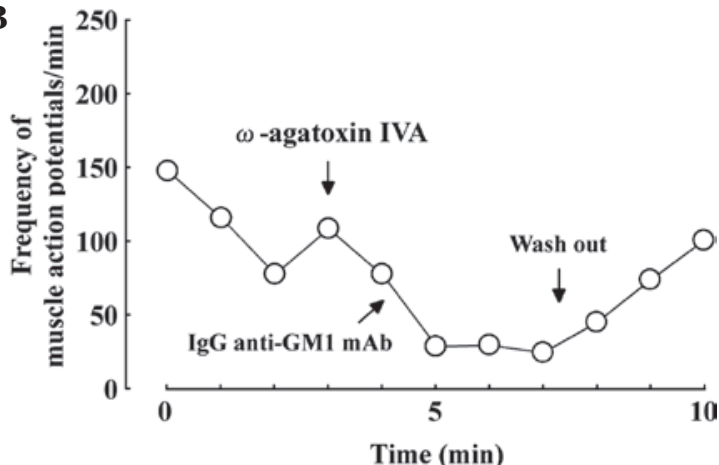

C

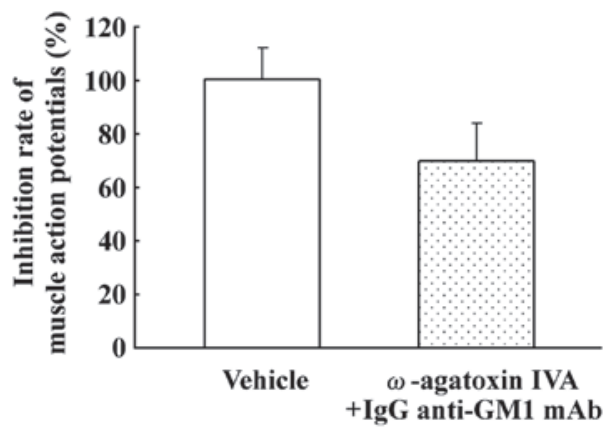

Figure 3. Effects of pretreatment with the P/Q-type calcium channel blocker, $\omega$-agatoxin IVA, on the inhibitory effect of IgG anti-GM1 mAb (1:100) on spontaneous muscle action potentials (SMAPs) in the spinal cord-muscle co-culture system. (A) Inhibition of SMAPs by pretreatment with $\omega$-agatoxin IVA $(10 \mathrm{nM})$ in the spinal cord-muscle co-cultures. (B) Time course showing the partial reversal by pretreatment with $\omega$-agatoxin IVA (10 nM) on the inhibitory effect on SMAPs mediated by IgG anti-GM1 mAb after washing. The number of SMAPs was measured every 5 sec. Arrows show the time of administration of $\omega$-agatoxin IVA, IgG anti-GM1 mAb, and exchange of the co-culture medium. (C) Inhibition rate of SMAPs following the addition of IgG anti-GM1 mAb. Error bars represent the mean \pm standard error of the mean of data pooled from 4-6 spinal cord-muscle co-cultures. mAB, monoclonal antibody.

A

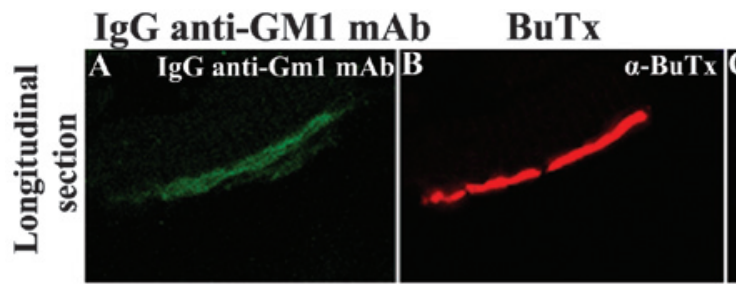

a 1A
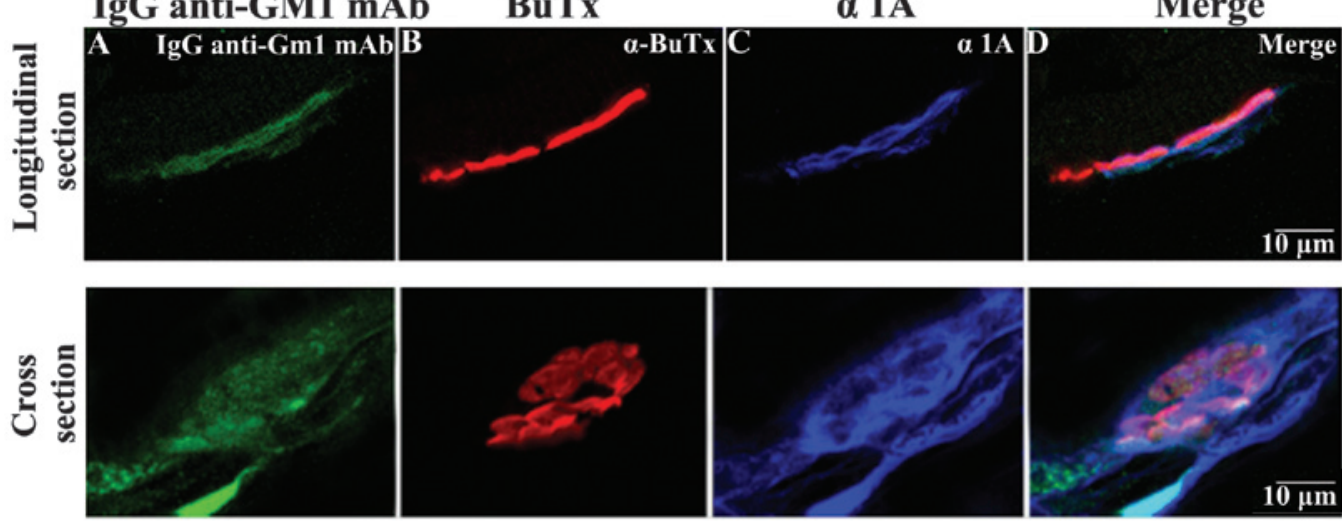

B

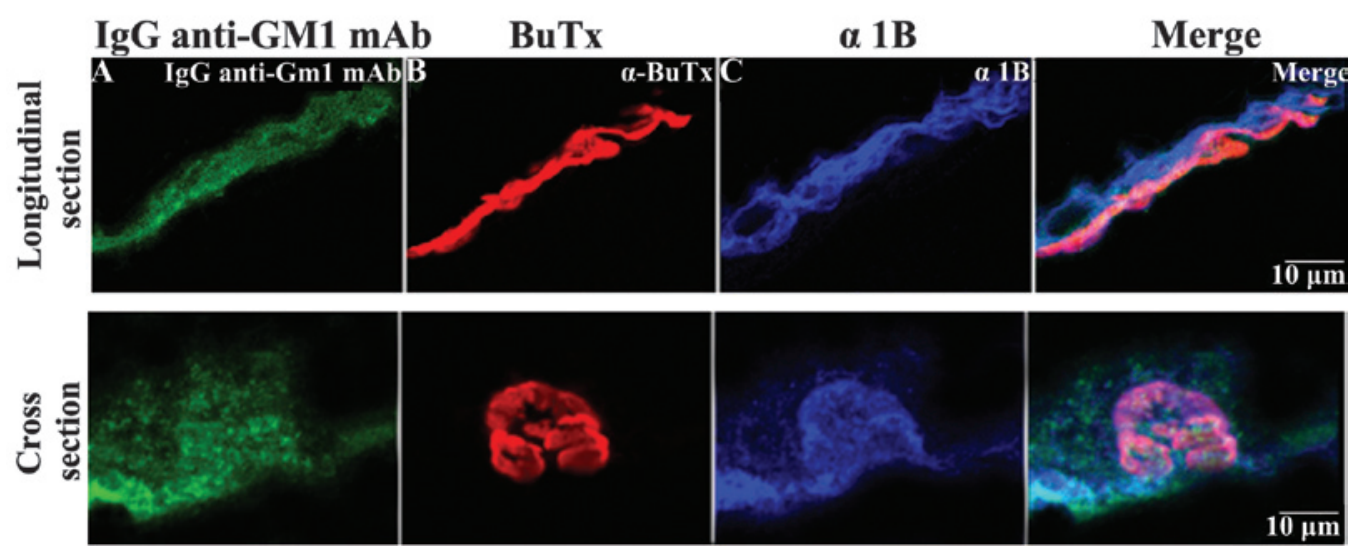

Figure 4. Immunostaining with IgG anti-GM1 mAb and calcium channel antibodies in cross-sections and longitudinal sections of the rat hemidiaphragms. (A) Staining with IgG anti-GM1 mAb (green) overlapped with the postsynaptic acetylcholine receptor cluster marker, $\alpha$-BuTx (red), and the anti-Ca $2.1\left(\alpha_{1 \mathrm{~A}}\right.$; 1:1,000; P/Q-type calcium channel; blue) antibody at the nerve terminal. (B) Staining with IgG anti-GM1 mAb (green) overlapped with $\alpha$-BuTx (red) and the anti- $\mathrm{Ca}_{\mathrm{v}} 2.2\left(\alpha_{1 \mathrm{~B}} ; 1: 1,000 ; \mathrm{N}\right.$-type calcium channel; blue) antibody. mAB, monoclonal antibody; $\alpha$-BuTx; $\alpha$-bungarotoxin. 
rapidly reduced the number of SMAPs at the NMJs within 1 min (Fig. 1A). Subsequently, SMAPs at the NMJs were blocked completely (Fig. 1B). The inhibitory effect of the IgG anti-GM1 mAb was reversed by washing with the co-culture medium. Thus, IgG anti-GM1 mAb significantly reduced the rate of SMAPs at the NMJs $(n=4,70.8 \pm 4.8 \%$; Fig. $1 C)$.

Effects of pretreatment with $\omega$-conotoxin GVIA on SMAPs in the spinal cord-muscle co-culture system. Fig. 2 shows the effect of pretreatment with the N-type calcium channel blocker, $\omega$-conotoxin GVIA (30 nM), and IgG anti-GM1 mAb on the SMAPs. The inhibitory effect of IgG anti-GM1 mAb was completely blocked by pretreatment with $\omega$-conotoxin GVIA in the spinal cord-muscle co-culture systems (Fig. 2A). Fig. 2B shows the time course of the reversal of the IgG anti-GM1 mAb-induced inhibition of SMAPs by pretreatment with $\omega$-conotoxin GVIA. Thus, pretreatment with $\omega$-conotoxin GVIA attenuated the inhibitory effect on SMAPs induced by IgG anti-GM1 mAb (Fig. 2C).

Effects of $\omega$-agatoxin IVA on SMAPs in the spinal cord-muscle co-culture system. Fig. 3 shows the effect of the P/Q-type calcium channel blocker, $\omega$-agatoxin IVA $(10 \mathrm{nM})$, and IgG anti-GM1 mAb on SMAPs. $\omega$-agatoxin IVA slightly decreased the frequency of SMAPs in the spinal cord-muscle co-culture systems (Fig. 3A). In addition, the inhibitory effect of the IgG anti-GM1 mAb was partially blocked by pretreatment with $\omega$-agatoxin IVA in spinal cord-muscle co-cultures. Fig. 3B shows the time course of the partial reversal of the inhibitory effect on SMAPs produced by IgG anti-GM1 mAb following pretreatment with $\omega$-agatoxin IVA. Therefore, pretreatment with $\omega$-agatoxin IVA slightly attenuated the inhibitory effect on SMAPs induced by IgG anti-GM1 mAb (Fig. 3C).

Immunohistochemical staining using IgG anti-GM1 mAb and calcium channel antibodies in hemidiaphragm sections. The localization of IgG anti-GM1 mAb and various calcium channels in the nerve terminal was determined using immunohistochemistry. As shown in Fig. 4A, the rat hemidiaphragm sections were found to stain positively for the anti-Ca $2.1\left(\alpha_{1 \mathrm{~A}}\right.$; P/Q-type calcium channel; 1:1,000; blue) antibody, and the staining overlapped partially with the IgG anti-GM1 mAb staining (green) and $\alpha$-BuTx staining (red). In addition, anti-Ca $2.2\left(\alpha_{1 \mathrm{~B}}\right.$; N-type calcium channel; $1: 1,000$; blue) antibody staining overlapped with IgG anti-GM1 mAb staining and $\alpha$-BuTx staining, similarly to the anti- $\alpha_{1 \mathrm{~A}}$ antibody staining (Fig. 4B). Thus, IgG anti-GM1 mAb binding was localized at the motor nerve terminal, and the staining corresponded with $\mathrm{N}$-type calcium channels at the motor nerve terminal and partially overlapped with $\mathrm{P} / \mathrm{Q}$-type calcium channel staining.

\section{Discussion}

The results of the present study indicated that the inhibitory effects on SMAPs induced by IgG anti-GM1 mAb were completely blocked by pretreatment with $\omega$-conotoxin GVIA, an N-type calcium channel blocker, in spinal cord-muscle co-culture systems. However, pretreatment with the P/Q-type calcium channel blocker, $\omega$-agatoxin IVA, only partially blocked the IgG anti-GM1 mAb-induced inhibition of SMAPs.
Previous studies have demonstrated that calcium channels are targets for anti-ganglioside antibody-mediated attack $(11,12)$; however, the mechanism through which the IgG anti-GM1 mAb inhibits neurotransmitter release and the activity of calcium channels has not been fully clarified. Buchwald et al (13) and Ortiz et al (14) proposed that the sera of patients with GBS may block calcium channels located on axon terminals. In addition, a number of studies have indicated that calcium ion influx via VDCCs triggers the release of acetylcholine from motor nerve terminals at NMJs (15-17). Furthermore, prior incubation with $\omega$-agatoxin IVA has been shown to completely block the inhibitory effect of IgM mAbs against GM2, GalNAc-GD1a and GalNAc-GM1b on neurotransmitter release (14). Thus, the present results indicate that the IgG anti-GM1 mAb blocks calcium influx via N-type and P/Q-type calcium channels. In addition, the IgG anti-GM1 mAb antibody was previously demonstrated to inhibit VDCC currents (6). The present data may be explained by the involvement of VDCC currents in the effects of IgG anti-GM1 mAb on neurotransmitter release.

IgG anti-GM1 mAb staining was observed to overlap with $\mathrm{P} / \mathrm{Q}$-type and N-type calcium channel staining, indicating that the IgG anti-GM1 mAb binds to the two types of calcium channels at the motor nerve terminal of the rat hemidiaphragm. GM1, GD1 and GD1b ganglioside epitopes exist in presynaptic membranes or the nodal region, which is crucial for peripheral nerve transmission. Previous studies have demonstrated the rapid uptake of anti-ganglioside antibodies at the presynaptic motor nerve terminal, as compared with the axolemmal membrane at the node of Ranvier $(18,19)$. Thus, P/Q-type and $\mathrm{N}$-type calcium channels are implicated as targets for autoantibodies in patients with GBS. However, GBS is associated with antibodies against numerous gangliosides, including GM1b, GD1a, GD1b, GalNAc-GD1a and GQ1b; thus, whether SMAPs are additionally inhibited by other anti-ganglioside antibodies is yet to be determined.

In conclusion, the results of the present study demonstrated that the binding site for the IgG anti-GM1 mAb is present on $\mathrm{P} / \mathrm{Q}$-type and N-type calcium channels in the nerve terminals of NMJs. Thus, IgG anti-GM1 antibodies may be among the factors that result in muscle weakness in patients with GBS through binding to calcium channels.

\section{Acknowledgements}

This study was supported in part by a grant from the Japan Society for the Promotion of Science Grants-in-Aid for Scientific Research (no. 22590087).

\section{References}

1. Buchwald B, Toyka KV, Zielasek J, Weishaupt A, Schweiger S and Dudel J: Neuromuscular blockade by $\mathrm{IgG}$ antibodies from patients with Guillain-Barré syndrome: A macro-patch-clamp study. Ann Neurol 44: 913-922, 1998.

2. O'Hanlon GM, Bullens RW, Plomp JJ and Willison HJ: Complex gangliosides as autoantibody targets at the neuromuscular junction in Miller Fisher syndrome: A current perspective. Neurochem Res 27: 697-709, 2002.

3. Taguchi K, Ren J, Utsunomiya I, Aoyagi H, Fujita N, Ariga T, Miyatake T and Yoshino H: Neurophysiological and immunohistochemical studies on Guillain-Barre syndrome with IgG anti-GalNAc-GD1a antibodies - effects on neuromuscular transmission. J Neurol Sci 225: 91-98, 2004. 
4. Quattrini A, Lorenzetti I, Sciorati C, Corbo M, Previtali SC, Feltri ML, Canal N, Wrabetz L, Nemni R and Clementi E: Human IgM anti-GM1 autoantibodies modulate intracellular calcium homeostasis in neuroblastoma cells. J Neuroimmunol 114: 213-219, 2001.

5. Hotta S, Nagaoka T, Taguchi K, Nakatani Y, Utsnomiya I, Masuda Y, Abe K and Yuki N: Neurophysiological and immunohistochemical studies of IgG anti-GM1 monoclonal antibody on neuromuscular transmission: Effects in rat neuromuscular junctions. Neurol Sci 35: 205-213, 2014.

6. Nakatani Y, Hotta S, Utsunomiya I, Tanaka K, Hoshi K, Ariga T, Yu RK, Miyatake T and Taguchi K: Cav2.1 voltage-dependent $\mathrm{Ca}^{2+}$ channel current is inhibited by serum from select patients with Guillain-Barré syndrome. Neurochem Res 34: 149-157, 2009.

7. Davies A, Douglas L, Hendrich J, Wratten J, Tran Van Minh A, Foucault I, Koch D, Pratt WS, Saibil HR and Dolphin AC: The calcium channel alpha2delta-2 subunit partitions with CaV2.1 into lipid rafts in cerebellum: Implications for localization and function. J Neurosci 26: 8748-8757, 2006.

8. Taverna E, Saba E, Rowe J, Francolini M, Clementi F and Rosa P: Role of lipid microdomains in P/Q-type calcium channel (Cav2.1) clustering and function in presynaptic membranes. J Biol Chem 279: 5127-5134, 2004.

9. Nakatani Y, Nagaoka T, Hotta S, Utsunomiya I, Yoshino H, Miyatake T, Hoshi K and Taguchi K: IgG anti-GalNAc-GDla antibody inhibits the voltage-dependent calcium channel currents in PC12 pheochromocytoma cells. Exp Neurol 204 380-386, 2007.

10. Taguchi K, Shiina M, Shibata K, Utsunomiya I and Miyatake T: Spontaneous muscle action potentials are blocked by N-type and $\mathrm{P} / \mathrm{Q}$-calcium channels blockers in the rat spinal cord-muscle co-culture system. Brain Res 1034: 62-70, 2005.
11. Tanaka Y, Waki H, Kon K and Ando S: Gangliosides enhance $\mathrm{KCl}$-induced $\mathrm{Ca}^{2+}$ influx and acetylcholine release in brain synaptosomes. Neuroreport 8: 2203-2207, 1997.

12. Ledeen RW and Wu G: Ganglioside function in calcium homeostasis and signaling. Neurochem Res 27: 637-647, 2002.

13. Buchwald B, Zhang G, Vogt-Eisele AK, Zhang W, Ahangari R, Griffin JW, Hatt H, Toyka KV and Sheikh KA: Anti-ganglioside antibodies alter presynaptic release and calcium influx. Neurobiol Dis 28: 113-121, 2007.

14. Ortiz N, Rosa R, Gallardo E, Illa I, Tomas J, Aubry J, Sabater M and Santafé M: IgM monoclonal antibody against terminal moiety of GM2, GalNAc-GD1a and GalNAc-GM1b from a pure motor chronic demyelinating polyneuropathy patient: Effects on neurotransmitter release. J Neuroimmunol 119: 114-123, 2001.

15. Iwasaki S, Momiyama A, Uchitel OD and Takahashi T: Developmental changes in calcium channel types mediating central synaptic transmission. J Neurosci 20: 59-65, 2000.

16. Pagani R, Song M, McEnery M, Qin N, Tsien RW, Toro L, Stefani E and Uchitel OD: Differential expression of alpha 1 and beta subunits of voltage dependent $\mathrm{Ca}^{2+}$ channel at the neuromuscular junction of normal and P/Q $\mathrm{Ca}^{2+}$ channel knockout mouse. Neuroscience 123: 75-85, 2004.

17. Urbano FJ, Pagani MR and Uchitel OD: Calcium channels, neuromuscular synaptic transmission and neurological diseases. J Neuroimmunol 201-202: 136-144, 2008.

18. Fewou SN, Rupp A, Nickolay LE, Carrick K, Greenshields KN, Pediani J, Plomp JJ and Willison HJ: Anti-ganglioside antibody internalization attenuates motor nerve terminal injury in a mouse model of acute motor axonal neuropathy. J Clin Invest 122: 1037-1051, 2012.

19. Fewou SN, Plomp JJ and Willison HJ: The pre-synaptic motor nerve terminal as a site for antibody-mediated neurotoxicity in autoimmune neuropathies and synaptopathies. J Anat 224: 36-44, 2014. 